\title{
ÉDUCATION ET MODERNISATION EN RUSSIE TSARISTE, 1861-1917
}

\author{
Jean-Guy Lalande
}

Eklof, Ben. Russian Peasant Schools: Officialdom, Village Culture, and Popular Pedagogy, 1861-1914. Berkeley: University of California Press, 1986. Pp. xv, 652.

Brooks, Jeffrey. When Russia Learned to Read: Literacy and Popular Literature, 1861-1917. Princeton: Princeton University Press, 1985. Pp. xxii, 450.

Krukones, James H. To the People: The Russian Government and the Newspaper "Sel' skii vestnik" ("Village Herald"), 1881-1917. New York: Garland Publishing, 1987. Pp. ix, 279.

Otto, Robert. Publishing for the People: The Firm Posrednik, 1885-1905. New York: Garland Publishing, 1987. Pp. 251.

Seregny, Scott J. Russian Teachers and Peasant Revolution: The Politics of Education in 1905. Bloomington: Indiana University Press, 1989. Pp. x, 292.

Les cinquante demières années du régime tsariste en Russie continuent de susciter beaucoup d'intérêt parmi les historiens. La période est évidemment décisive: elle s'étend de l'abolition du servage à l'effondrement de la dynastie des Romanovs et elle témoigne des efforts faits par un gouvernement, soucieux de conserver au pays son statut de grande puissance, en vue du moderniser l'ensemble de la nation. L'alphabétisation et l'acculturation des masses populaires représentent un élément important de ce processus de modernisation. Une recension de cinq livres récents consacrés à l'étude de cette question devrait nous aider à mesurer, en partie, la nature des défis à relever et l'ampleur des succès atteints.

Dès la première page de ce très gros livre qu'est Russian Peasant Schools, l'auteur s'inscrit en faux contre l'image traditionnelle d'une paysannerie russe naïve, impulsive, irrationnelle, ignorante, routinière et qui, conséquemment, «needed stern guidance» (p. 1). La paysannerie d'Eklof en est une qui, au contraire, sait découvrir quels sont ses intérêts vitaux et qui agit de façon rationnelle. Ainsi, face à l'indifférence des élites dirigeantes, les paysans prennent eux-mêmes l'initiative de bâtir et d'entretenir, à leurs frais, un certain 
nombre d'ecoles. Effort louable, certes, mais nettement insuffisant! Trente ans après l'émancipation des serfs en 1861, le gouvernement central, réalisant que le maintien du statut de grande puissance pour la Russie est directement relié à l'enclenchement du processus de modernisation de l'économie et de la société, intervient de façon décisive: il le fait d'abord par l'intermédiaire des zemstva (ces organes de gouvernement local avec lesquels, du reste, il collabore assez bien), puis, à partir de 1908, du ministère de l'éducation, lequel s'engage à mettre en place progressivement un système d'éducation primaire à l'échelle du pays. $\grave{A}$ la veille de la première guerre mondiale, note Eklof, l'empire des tsars «was well on its way to achieving full universal primary education» (p. 286); seules les régions éloignées à faible densité de population restent alors une terre en friche.

Mais quel est le profil des enseignants russes? Le tableau est plutôt sombre: dans l'ensemble, ils sont relativement bien préparés à remplir leurs fonctions, jeunes, généralement célibataires, de sexe féminin (surtout à partir des années 1880) et très mobiles; sous-payés (il doit même, à l'occasion, quêter son salaire), leur vie personnelle soumise à l'oeil vigilant et parfois inquisiteur du zemstvo ou de l'inspecteur, ils sont dépourvus d'à peu près tout support intellectuel et psychologique (accès aux livres, cours de rattrapage, congrès); sans grand statut social, incapables de se faire accepter et reconnaitre à leur juste valeur, ils ne parviennent donc pas à s'enraciner dans le village-largement en raison de la méfiance, voire de l'hostilitê de ses habitants-et, conséquemment, ils ne réussissent que rarement à leur transmettre des idées ou des valeurs nouvelles. Bref, de conclure Eklof, ces étrangers vivent une situation de perpétuelle aliénation: "Teachers were, in a sense, 'contaminated'....The 'contamination' came from contact with children and peasants, which lowered the status of teachers in the eyes of the elite, and from contact with the elite, which polluted teachers in the eyes of the peasants» (p. 481).

Dans la troisième partie de son livre, Eklof explique le pourquoi d'un tel état de faits: c'est que les paysans utilisent, ă leurs fins, les enseignants. En effet, le paysan sait exactement ce qu'il veut pour son enfant (essentiellement le garçon, l'idée d'éduquer la fille ne présentant guère d'avantages): qu'il apprenne à lire, à écrire et à compter et non à développer une certaine curiosité et/ou un sens critique par rapport à son milieu ambiant; un savoir trop élaboré, un contact avec la culture de l'élite russe représentent une dépense inutile et risquent d'altérer ultérieurement les habitudes de travail de l'enfant, voire de miner l'autorité patriarcale à l'intérieur de la cellule familiale. Le rythme et l'intensité du calendrier scolaire se plient aux mêmes impératifs: ainsi, plutôt que de chercher à imposer un ordre nouveau, l'école russe adapte son horaire au caractère saisonnier de la vie au village. Concrètement, cela veut dire que l'année scolaire, plus courte à la campagne qu'à la ville, fluctuant considérablement de province à province (le sol russe n'est pas également fertile), commence tard (après la moisson) et se termine tôt (avant les semences) et que, si la fréquentation scolaire est remarquablement élevée (près de 90\%), elle est de courte durée: très peu fréquentent l'école pendant plus de deux ans et environ un élève sur dix complète 
le programme d'études primaires d'une durée de trois ans. Mais pour le paysan moyen, ce taux d'abandon, qu'Eklof relie ni à la paresse, l'ignorance ou la pauvreté du paysan, ni même à l'attrait d'un revenu supplémentaire qu'amènerait le travail de l'enfant aux champs ou à l'usine, n'a rien de tragique puisque l'essentiel est acquis, soit la maîtrise d'un certain nombre d'habiletés techniques.

Dans la demière partie de l'ouvrage, l'auteur essaie d'évaluer les résultats d'un tel enseignement. Appuyée sur les rapports et observations d'inspecteurs, d'enseignants et de zemsiva, de même que sur les résultats d'examens donnés à d'anciens élèves, sa conclusion découle naturellement de ce que précède: les elèves peuvent lire (sans toujours comprendre, cependant), Ecrire et compter, mais seulement «within the limits prescribed by the needs of the family and village» (p. 418). Même l'obtention d'un diplôme ne modifie pas sensiblement les données: les diplômés restent à la campagne, très peu d'entre eux poursuivant leurs études aux niveaux secondaire et universitaire.

Fruit de recherches minutieuses utilisant tant des sources primaires que secondaires, truffé de données statistiques pertinentes, peut-être un peu long par moments, cet ouvrage rédigé en une langue claire a le grand mérite d'innover-à la fois en comparant l'expérience russe avec l'européenne et l'américaine et, davantage, en examinant l'école comme élément constitutif de la culture paysanne et comme reflet de la vie du village russe. De plus, en montrant comment les paysans on su tirer de l'école autre chose que ce que les élites aspiraient à leur transmettre, Eklof récuse l'approche classique qui fait de l'école une institution imposée, par décision gouvernementale, à une population croupissant dans son ignorance. S'il reste encore certaines questions à élucider-le poids des politiques de russification auprès des multiples nationalités non-russes de l'empire; le rôle joué par l'armée en tant qu'agent d'alphabétisation; l'impact de la scolarisation sur les courants migratoires entre villes et campagnes; les manuels utilisés; la signification du processus de féminisation du corps enseignant-, l'étude de Ben Eklof apporte un nouvel éclairage à l'histoire de l'enseignement primaire en Russie durant les cinquante dernières années du régime tsariste.

Mais comment les paysans utiliseront-ils ce nouvel outil? Que liront-ils, en priorité? Dans un livre d'une qualité exceptionnelle intitulé When Russia Learned to Read, Jeffrey Brooks nous fournit d'intéressants éléments de réponse. L'auteur montre d'abord comment les paysans russes, émancipés depuis 1861 et réalisant à quel point une aptitude à lire pourrait leur être utile (à l'usine, à la ville, à l'armée, à l'église, en migration, face à l'appareil administratif), acquièrent, en plus grand nombre qu'auparavant, une éducation primaire. Une littérature à la fois plus diversifiée et plus vaste éclôt afin de satisfaire la curiosité et les goûts de ce nouveau public-lecteur. L'auteur décrit ensuite les formes multiples sous lesquelles cette litterature populaire-prolongement, en quelque sorte, de l'école-s'exprime: le loubok (petit livret d'une trentaine de pages environ alliant l'image au texte, vendu dans les villes et les campagnes et dont les thèmes, puisant jusque-là dans le folklore et les vies de saints, se sécularisent dans la 
second moitié du dix-neuvième siècle), les périodiques illustrés, les journaux quotidiens et les feuilles de choux («potboilers», p. 109).

L'originalité et le brio de cet ouvrage tiennent surtout aux quatre chapitres où Brooks procède à une étude du contenu de cette littérature populaire (complétée, à l'occasion, par des parallèles avec les littératures populaires française, anglaise et américaine) et établit une corrélation avec la réalité politique, économique, sociale et culturelle de la Russie d'alors. L'auteur retient, pour fins d'analyse, quatre thèmes majeurs: le bandit, ou l'iđée de liberté et d'ordre; nationalisme et identité nationale; science et superstition; succès. Chacun d'entre eux est introduit à l'aide de résumés d'intrigues, parfois doublés d'illustrations absolument fascinantes. En voice quelques exemples. Le bandit, s'il est libre, reste un homme condamné, violent et sanguinaire, privé de tout confort familial et communautaire et qui ne peut, à moins qu' il ne préfere l'humiliation et la mort en solitaire, réintégrer les rangs de la société que s'il se rachète «through state service» (p. 190)-rite essentiel pour bien illustrer que «the Russian act of repentance and redemption conforms to the logic of a society in which all political authority stems from a monarch» (p. 199). Or, au début du vingtième siècle, à un moment où les histoires de crimes gagnent en popularité, la communauté se substitue à l'État «in the salvation of outcast heroes» (p. 205); dans la même veine, le détective privé, nouveau représentant de l'ordre, joue le rôle de défenseur de la société civile et non de l'État. Brooks reconnaît, toutefois, que la transition n'est pas encore complète, anciennes et nouvelles mentalités se chevauchant: ainsi, ces nouveaux types de héros, maintenant capables «to enjoy freedom without paying a terrible price for rebellion» (p. 211), évoluent en terre étrangère. S'agit-il d'un simple caprice, fruit de l'imagination de l'auteur, ou plutôt d'un reflet implicite des transformations majeures (notamment l'affaiblissement de l'autocratie) opérées en Russie, suite à la révolution de 1905 ? L'auteur penche nettement du côté de la seconde hypothèse. Autre exemple intéressant parce que révélateur de ce même processus de modernisation: à nouveau, au début du vingtième siècle, aux symboles traditionnels-tsar et Église orthodoxe, dénigrement de l'étranger et du non-Russe-qui, jusque-1à, servaient à définir l'identité nationale russe, se substituent de nouveaux éléments-les vastes étendues de l'empire, la diversité des peuples qui l'habitent, la place des Grands Russes parmi eux-qui témoignent d'une indéniable sécularisation et d'un certain cosmopolitisme. Dernier exemple, enfin, où Brooks relie l'importance et la popularité du thème du succès dans la littérature populaire au fait que cette dernière apparaît à un moment où les structures de la société russe deviennent plus fluides, où les obstacles à l'initiative et à la mobilité s'abaissent et où se développe un auditoire de gens qui aspirent à une vie meilleure. L'auteur croit déceler, dans ces multiples images du succès et cette énumération des qualités (énergie, persévérance, ingéniosité, cran, impatience) qui en sont le gage, l'émergence d'un nouvel individualisme.

Le dernier chapitre traite de la réponse du gouvernement et des élites intellectuelles à cette efflorescence de littérature populaire. Le premier, pour qui 
Ia loyauté au tsar et à l'Église orthodoxe doit constituer la pierre angulaire de toute bonne oeuvre littéraire, s'efforce de réglementer, par la censure, les ouvrages indésirables et, de façon plus positive, de subventionner la publication d'oeuvres prêchant les valeurs traditionnelles (patriotisme et piété); l'objectif visé ici est de prévenir la corruption tant politique que morale des classes populaires. Quant aux secondes, leur réponse est unanime: quelle que soit leur orientation politique-slavophile, populiste, occidentaliste, libérale ou marxiste-, la littérature du loubok leur est tout à fait inacceptable; aussi cherchentelles à substituer à ce matérialisme vulgaire, à cette recherche du sensationalisme, une nourriture plus saine, plus éducative. Une telle approche, note Brooks, témoigne d'un certain impérialisme culturel dans la mesure où, convaincue «that the muzhik [paysan] was not competent to choose his own reading material» (p. 321 ), cette élite cherche, par souci de recréer une même identité culturelle, à imposer au simple lecteur, urbain ou rural, le genre et le contenu idéologique de ses lectures. Bien que le succès réel de cette littérature populaire donne la mesure de l'échec des efforts de l'élite cultivée, cette recherche d'une seule et même culture partagée par tous refera surface sous les Bolchéviques, ces derniers étant convaincus «that cultural choices could be curtailed in the name of high ideals» (p. 356).

Brooks conclue sa magistrale étude en soutenant que le simple paysan ou ouvrier russe, en exprimant son intérêt et sa préférence pour cette littérature populaire commerciale, s'est, après 1861 , plus facilement adapté aux changements politiques, économiques et sociaux que certains autres groupes, en particulier les enseignants et, surtout, les membres de l'intelligentsia. Une telle hypothèse, bien que séduisante, n'est évidemment pas facile à vérifier, elle me semble, en outre, assez discutable. Si l'intérêt populaire-sans doute attisé par le fait qu'écrivains et éditeurs, parce qu'issus tout récemment de ce même milieu social, connaissent les goûts de ce nouveau public-lecteur-est indéniable, une question demeure: dans quelle mesure une telle quête de l'imprimé ne constituet-elle pas un agréable moyen de s'évader du réel, de se transporter un instant dans un monde imaginaire afin de mieux oublier le terrible quotidien russe plutôt qu'un effort conscient d'y puiser de nouvelles règles de vie? Si tout lecteur ne partage pas nécessairement les idées et les valeurs qui peuvent agréablement meubler ses heures de lecture, n'est-il pas un peu périlleux d'extrapoler ainsi une Weltanschauung à partir de simples intrigues et thèmes littéraires? Qui plus est, l'histoire de la Russic au toumant du siècle semble justifier pleinement de telles réserves: en effet, comment réconcilier cette nouvelle identité nationale, cette fierté d'être Russe avec l'effondrement total du pays lors de la première guerre mondiale? Comment ne pas rappeler également la vigueur de l'appui populaire en faveur de la commune paysanne au moment même où Stolypine, en cherchant à provoquer son démembrement, privilégiait l'initiative individuelle, le succès matériel, la mobilité sociale? Cette solidarité collective des masses, ne fera-t-elle pas irruption de façon décisive durant l'année 1917 ? Enfin, ne serait-il pas 
approprié de distinguer entre public-lecteur de la ville et public-lecteur de la campagne? L'impact n'a certainement pas été le même dans l'un et l'autre cas!

Ce livre de Jeffrey Brooks, neuf, audacieux, tout à fait remarquable, a le très grand mérite d'ouvrir de nouvelles pistes de recherche et, s'il ne peut établir de façon définitive l'étendue et la profondeur des changements de mentalités, il remet en question - et de façon fort convaincante-un certain traditionalisme généralement attribué aux masses russes à la veille de la guerre et de la Révolution.

Les deux ouvrages suivants-ceux de Krukones et d'Oto-analysent en đétail les réponses des autorités gouvernementales et d'une certaine élite cultivée à ce nouveau phénomène de la littérature populaire commerciale.

Dans la foulée de l'assassinat d'Alexandre II en 1881, le gouvernement russe, inquiet des répercussions qu'un tel événement pourrait avoir sur la mentalité et le comportement des paysans, décide de publier un journal qui, tout en contrant l'influence des révolutionnaires, atteindrait directement les masses rurales.

Dans son livre, To the People..., James H. Krukones relate la nature de cette initiative gouvernementale et en évalue la portée. La partie la plus intéressante de cet ouvrage est certainement celle qui analyse le contenu du Sel'skii vestnik. Les principaux thèmes retenus par l'équipe éditoriale reflètent naturellement les préoccupations et les orientations idéologiques du régime tsariste: vies de saints et sermons illustrant l'idée que «whatever happened had happened because God had ordained it» (p. 73); la famille impériale des Romanovs, indispensable au bon fonctionnement du royaume; les lois et decrets du gouvernement, particulièrement ceux affectant les paysans (ainsi, à partir de 1906, la réforme de Stolypine occupe une place importante); l'agriculture (conseils pratiques de toutes sortes, nouvelles des marchés, démentis de la rumeur d'une éventuelle répartition des terres, importance, jusqu'en 1905-6, de la commune paysanne) et les métiers; les questions de politique extérieure et le patriotisme (thèmes particulièrement importants au moment de la guerre contre le Japon en 1904-5 et de celle contre les puissances centrales entre 1914 et 1917); les luttes politiques intemes: ainsi, des différents partis siégeant au parlement, le parti octobriste, sympathique à la monarchie, recueille la faveur du journal; un assortiment de sujets divers propres à la campagne: la prévention contre les feux et les maladies les plus répandues; l'alcoolisme, véritable fléau; la promotion du bon livre; l'anti-sémitisme, etc.

Dans quelle mesure les objectifs poursuivis ont-ils été atteints? Quelle influence cette vision moralisatrice de la réalité, telle que véhiculée par un organe gouvernemental de propagande, a-t-elle exercée sur le paysan russe? Bien qu'il ne soit pas facile de répondre à une telle question, reconnaît l'auteur, l'impact a certainement été réel et bénéfique, de même que l'appui en faveur du joumal (130 000 copies en 1905!). Néammoins, il importe de nuancer beaucoup, en raison de l'inefficacité du réseau de distribution du journal, du nombre finalement peu 
élevé de lecteurs et, sans doute davantage, de l'incapacité des hauts fonctionnaires du ministère de l'intérieur, parrains du Sel' skii vestnik, à bịen saisir et à accepter la différence entre censure et journalisme, même après une réorganisation du journal en 1905 . Tout à fait à l'aise avec la première, ils redoutent le second car il peut «stimulate curiosity, promote skepticism, breed discontent, foment rebellion» (p. 247). Une approche aussi audacieuse était évidemment tout à fait inacceptable pour un régime aussi conservateur et paternaliste que celui des Romanovs.

Ironie de l'histoire, cependant, les bureaux du Sel' skii vestnik, mis au rancart après le coup d'état bolchévique d'octobre 1917, allaient bientôt abriter le jounal Pravda! Une tout autre conception de l'éducation des masses et de la propagande allait s'imposer pour plusieurs décennies...

Au dix-neuvième siècle, des membres de l'intelligentsia russe, conscients que leur haut niveau de culture n'a été rendu possible que grâce au dur labeur du peuple, désirent lui rembourser une partie de cette dette. Deux possibilités s'offrent à eux: la lutte révolutionnaire en vue de renverser le régime ou un patient travail d'éduction mené auprès de la paysannerie. Dans son livre Publishing for the People, Robert Otto s'intéresse à cette dernière approche. L'auteur décrit le désir de cette intelligentsia modérée de mettre entre les mains du paysan un bon livre. L'objectif poursuivi est double: rétrécir sensiblement l'écart culturel qui la sépare du peuple et offrir à celui-ci autre chose que cette superficielle loubochnaya literatura. Cette intelligentsia est convaincue que le peuple ne s'interesse à ces denrées avariées que parce qu'il n'y a rien d'autre de disponible et que si une alternative valable existait, le peuple répudierait une telle littérature.

Les premières tentatives, animées par un généreux idéalisme, se soldent par un échec: «more was published about publishing for the people than was actually published for the people» (p. 32), explique l'auteur. Le projet, cependant, ne meurt pas. Posrednik (terme russe qui veut dire «Intermédiaire») sera finalement fondé en 1884, suite à la rencontre de l'écrivain L. Tolstoi et d'un de ses disciples, V.G. Chertkov, et grâce à la précieuse collaboration d'Ivan Sytin, à la fois financier, conseiller pour la mise en marché, éditeur et distributeur de Posrednik. Entre 1885 et 1905, Posrednik publiera environ 600 livres pour le peuple, rédigés par différents collaborateurs: auteurs établis, activistes sociaux travaillant dans les milieux d'éducation populaire, membres de l'intelligentsia et même, des paysans. Le message véhiculé par Posrednik est facilement identifiable: à un ensemble de conseils pratiques et de connaissances générales (géographie, sciences naturelles, médicine, économie) s'ajoute-et c'est là la raison d'être de toute l'entreprise -une Weltanschauung tolstö̈enne, c'est-à-dire un appel à lutter contre le mal à l'intérieur de soi et à cultiver les vertus de patience, de douceur, de pitié, de pardon, de sacrifice, d'humilité et d'écoute de la voix de Dieu. Curieusement, une telle approche moralisatrice est prise à partie par l'Église orthodoxe russe qui redoute l'influence, jugée néfaste, de Tolstoi; par contre, elle 
est bien reçue des paysans, parfois même bouleversés jusqu'aux larmes, et de membres de l'intelligentsia, encore que certains d'entre eux remettent en question l'utilité de prêcher à une population aussi opprimée, sur le plan matériel, l'humilité et la résignation. En conclusion, Otto essaie d'évaluer dans quelle mesure l'objectif poursuivi a été atteint. Le verdict reste partagé: si l'impact est indéniable auprès du peuple, si l'altruisme de l'intelligentsia est réel, l'auteur souligne qu'en dépit de tous ces efforts la loubochnaya literatura est restée très présente dans la vie du peuple. Malheureusement ce livre, ou plutôt cette thèse de doctorat faisant partie de la "Garland Series of Outstanding Dissertations», est d'une lecture rarement agréable, tellement le texte est parsemé d'un nombre incalculable de fautes typographiques!

Dans un livre très fouillê et très dense, Russian Teachers and Peasant Revolution, Scott $J$. Seregny reprend certains thèmes développés par Eklor: le développement d'un système scolaire de niveau élémentaire; le profil de l'enseignant au primaire; les relations conflictuelles entre zemstva et gouvernement central; le désir de ce dernier d'encourager, par le perfectionnement des instituteurs et par l'alphabétisation des paysans, la modernisation du pays, tout en maintenant l'ordre à la campagne.

L'originalité de ce livre réside dans le portrait plus nuancé qu'il présente des enseignants et de leur rôle à la campagne. Précisant l'equisse de B. Eklof, Seregny montre comment, face aux politiques restrictives du gouvernement, une conscience professionnelle se développe peu à peu parmi le corps enseignant, durant les années qui précèdent la révolution de 1905 , et s'organise, d'abord au sein de sociétés d'aide mutuelle, puis, d'un véritable syndicat. Toutes ces initiatives créent une solidarité et des attentes nouvelles, de même qu'un haut niveau de frustration, lequel débouchera, en 1905, sur l'action politique. Certes, reconnaît l'auteur, un tel engagement n'est pas unanime: la majorité des enseignants, même en 1905, "eschewed politics and seemed barely aware of the pressing issues that concerned Russian educated society» (p. 208). Néammoins, les plus politisés d'entre eux, en dépit de la présence d'éléments réactionnaires (les Cent-Noirs), sortent de leur isolement et s'engagent résolument en faveur des paysans, soit en leur expliquant les nouvelles de politique interieure et extérieure rapportées dans les journaux, soit en aidant les communes rurales à rédiger des pétitions, soit encore (même lorsqu'abandonnés, après Octobre 1905, par une noblesse maintenant effarouchée) en aidant les paysans à organiser leurs propres syndicats. Si des enseignants agissent ainsi, soutient Seregny, c'est qu'ils se sont résolument identifiés aux demandes populaires en faveur de réformes politiques, économiques et sociales majeures (v.g. le partage des terres, l'assemblée constituante) et, par le fait même, qu'ils ont réussi à abattre les traditionnelles barrières d'incompréhension et de méfiance qui séparaient l'enseignant et le paysan. La dernière preuve de cette symbiose est l'élection d'enseignants du niveau primaire à titre de députés aux première et seconde Doumas de l'empire. Certes, de conclure l'auteur, la réaction inaugurée par 
Stolypine en 1906 interrompera brusquement les initiatives multiples de ces enseignants, mais cette victoire du gouvernement sera bien éphémère. Rappelant l'importante contribution politique apportée par l'enseignant allemand (soit la remarque de Bismarck à propos de la victoire militaire remportée à Sedan en 1870 ) et français (soit l'acceptation en province de la Troisième République), Seregny critique l'approche du gouvernement russe, puisque, en dernier ressort, elle ne parvint pas à créer «a confident cadre of professionals, commanding authority in the village and asserting official ideology and values. In fact, just the opposite occurred» (p. 208).

Que conclure? Quelques lecteurs de cette revue regretteront certainement qu'aucun de ces auteurs (la seule exception étant B. Eklof, et encore le fait-il de façon très périphérique) n'ait intégré leur étude à l'intérieur des grandes théories de l'éducation. Leur lecture n'en présente pas moins un indéniable intérêt.

Deux conclusions majeures se dégagent de la présente recension. La première concerne les classes populaires: s'écartant de l'interprétation stéréotypée et traditionnelle, Brooks et Eklof démontrent de façon convaincante comment celles-ci, loin d'être une pâte inerte et immobile qui accepte, sans sourciller, de se faire modeler par les élites intellectuelles ou gouvernementales, prennent, au contraire, conscience de leur propre identité et opposent à ces mêmes élites la légitimité de leurs aspirations et de leurs besoins. Surpris par une telle audace et un tel dynamisme, la gent cultivée et le gouvernement, sous des dehors peints aux couleurs de la générosité (soit l'idée d'élever le simple peuple à un plus haut niveau de culture) et de la reconnaissance (soit l'idée, puisée chez $P$. Lavrov, de la dette contractéc à l'endroit du peuple), s'efforceront de récupérer l'allégeance d'une paysanneric et d'une classe ouvrière qui leur semblent voguer, en pleine dérive, vers de périlleux récifs. Les recherches de Krukones et d'Otto, tout en notant la sincérité des aspirations de cette élite, illustrent clairement le caractère mitigé et parcellaire des succès remportés. La seconde conclusion découle de la première: au célèbre débat amorcé par Leopold H. Haimson, il y a maintenant plus de 25 ans, à propos des chances de survie d'un empire tsariste engagé dans un processus de modernisation accéléré, Seregny, pour un, appuie la thèse de la polarisation entre obshchestvo (société éduquée) et gouvernement central; quant aux quatre autres auteurs, en soulignant l'écart culturel existant entre les différentes composantes de la société russe, ils dévoilent à nouveau l'isolement, la fragilité et - l' l'école, le livre et le journal jouant davantage le rôle d'éveilleur des intelligences que de rempart de l'autocratie-la grande vulnérabilité du régime tsariste à l'aube du vingtième siècle. 